\section{Negatice Stiffness Vibration Isolation Technology for Nanotechnology}

\section{As Nanotech Applications Become More Diverse, the Need for Reliable Vibration Control Has Become Increasingly Critical}

\author{
David L. Platus \\ Minus K Technology, Inc., Inglewood, CA \\ david@minusk.com
}

It wasn't too long ago that making the decision where to locate your scanning probe microscope was a simple one put it in the basement where the ambient vibration was minimized. But recently, with nanotechnology applications growing exponentially, scientists and engineers are putting their equipment in a multitude of locations where vibration noise is significantly high. Scanning probe microscopes, interferometers and stylus profilers are being sited in locations that pose a serious challenge to vibration isolation.

Additionally, in an effort to keep their nano-equipment costs as low as possible by cutting out the peripherals, many academics and industries are not adequately providing for vibration isolation on their ultra-sensitive nano-equipment that they are putting into their facilities. Although high-budget installations (valued in the hundreds-of-thousands of dollars) typically incorporate adequate vibration isolation, this is not the case with many lesser-budget set-ups (those spending under $\$ 120,000$ for equipment), which represents the area of most rapid growth in the nanotechnology universe. It is estimated that $40 \%$ to $50 \%$ of these sites, in both academia and industry, are initiated with inadequate vibration isolation.

The above is influenced to some degree by the fact that those specifying nano-equipment do not always fully grasp the extreme sensitivity of the instruments, or that they require proper site selection and vibration isolation. With any type of microscope or other nano-instrument, even a high-powered optical microscope, you have got to put noise isolation there or you will end up with diffused and fuzzy imaging, and sometimes no image at all, resulting in reduced operability of a facility's nano-equipment.

Vibration isolators are one of those necessities that people are not really focused on when they go to purchase an instrument like an AFM (atomic force microscope). It is different with the bigger scanning electron microscopes and transmission electron microscopes; because you are dealing with a very expensive piece of gear that may technically need all sorts of mechanical isolation in order to work properly.

When you get into the smaller instruments like white light interferometers, laser interferometers, stylus profilers, and atomic force microscopes, then you get problems with site preparation. In many cases there is not a lot of site prep done, despite the fact that these instruments may be sitting on the fourth floor of a building, and without isolation will end up getting really poor images. They first have to solve their noise problem - and that means looking at some sort of mechanical isolation.

As instrumentation gets more and more complex, and measurements are being done at a smaller and smaller level, those vibrations that are present will start to dominate, and the need for more effective isolation increases. Isolators have been used since the beginning with atomic force microscopes in the 1980's, but there weren't that many AFMs then and most of them were in basements. The use of nano-instrumentation has grown dramatically, and the need for increased isolation has followed that trend.

The vibrations are usually very subtle. What you will not feel with your hands or feet will cause considerable noise and disturbance to an AFM or interferometer. The noise is caused by a multitude of things, it is not just originating from one spot. Every single building is making noise. Depending on how high up off the ground you are, and how old the building is, you are going to get a constant vibration. Within the building itself you have things that are going to create even more vibrations, such as the heating and ventilation system, fans, pumps that are not properly isolated, and elevators. These mechanical devices create a tremendous amount of vibration in the building, and depending on how far away the instruments are from it, they may or may not be adversely affected.

External to the building, your equipment may be influenced by vibrations from adjacent traffic, wind, construction, and other elements. These internal and external influences cause lower frequency vibrations, which raise havoc with nano-instrumentation. The wind blows and you get a little movement. Just the sway of it is around $2 \mathrm{~Hz}$, and causes a substantial resonance. A train near the building can cause movement in the cement slab, nothing that a viewer would ever feel, but for instrumentation purposes it has disastrous consequences. Imagine trying to measure a very few angstroms or nanometers of displacement. You have got to have an absolutely stable surface upon which to rest your instrument. If you don't, any of that vibration transferred into the mechanical structure of your instrument will cause vertical noise. And fundamentally, an inability to measure these kinds of high-resolution features.

An isolator is used to solve a problem, and how bad the problem is determines the solution you need. Since the 1960's air tables have been used for mechanical noise isolation. Basically cans of air, they are still the most popular isolators used. But, air tables with resonant frequencies at 2 to $2-1 / 2 \mathrm{~Hz}$ can typically only handle vibrations down to about 8 to $10 \mathrm{~Hz}$, not quite low enough for optimum performance with modern nano-equipment. For purposes of clarity in scanning probe microscopes and interferometers, air tables are an inefficient isolation solution. The air systems had been adequate up until a few years ago when better isolation was required.

As some may recall from the early years of nanotechnology, research scientists were fond of suspending their very expensive AFMs from bungee cords hanging from the ceiling, and sustained acceptable vibration isolation. Although some are still employing this technique, their numbers are dwindling-many aren't willing to take that risk any longer, and have switched over to other isolation systems.

One of these is active isolation, also known as electronic force cancellation. Active isolation uses electronics to sense the motion, and then puts in equal amounts of motion electronically to compensate, effectively canceling out the unwanted motion. Their efficiency is fine for application with the latest nanotechnology, as they can start isolating as low as $0.7 \mathrm{~Hz}$, quite sufficient for isolating the lower frequencies that are so damaging to image clarity with SPMs and interferometers.

However, if you can get things mechanically isolated without having to use energy, such as in the form of electricity, then you 


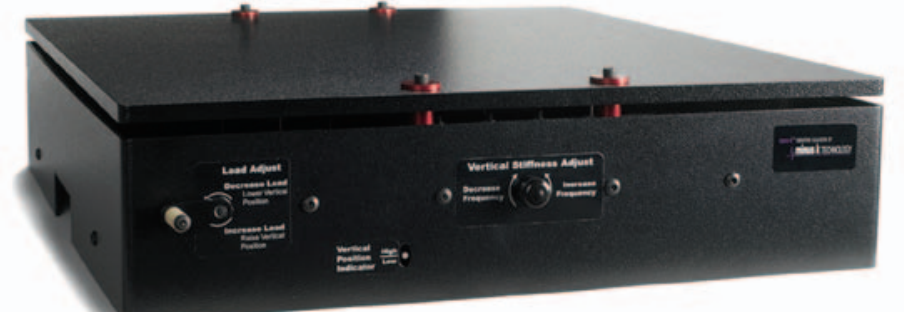

are inherently better off. If you don't have to have some form of supplied energy to run your isolator, then you will not be so negatively influenced by problems of electronic dysfunctions and power modulations, which can interrupt scanning.

Negative-stiffness vibration isolation systems have become a growing choice for nanotechnology applications. Not only are they a highly workable vibration solution, but their cost is significantly less - up to one-third the price of active systems-making it an economical solution to cost-conscious administrators.

This is a passive approach for achieving low vibration environments and isolation against sub-Hertz vibrations. These isolation systems enable vibration-sensitive instruments, such as scanning probe microscopes, micro-hardness testers and scanning electron microscopes to operate in severe vibration environments, such as upper floors of buildings and clean rooms. The images and data produced are many times better than those achievable with pneumatic isolators.

Negative-stiffness isolators employ a unique-and completely mechanical-concept in low-frequency vibration isolation. Vertical-motion isolation is provided by a stiff spring that supports a weight load, combined with a negative-stiffness mechanism (NSM). The net vertical stiffness is made very low without affecting the static load-supporting capability of the spring. Beam-columns connected in series with the vertical-motion isolator provide horizontal-motion isolation. The horizontal stiffness of the beam-columns is reduced by the "beam-column" effect. (A beam-column behaves as a spring combined with an NSM.) The result is a compact passive isolator capable of very low vertical and horizontal natural frequencies and very high internal structural frequencies. The isolators (adjusted to $1 / 2 \mathrm{~Hz}$ ) achieve $93 \%$ isolation efficiency at 2 $\mathrm{Hz} ; 99 \%$ at $5 \mathrm{~Hz}$; and $99.7 \%$ at $10 \mathrm{~Hz}$.

Improved vibration isolation directly correlates to improved instrument performance. When you are trying to measure atomic scale features, mechanically stable support structures are critically important. Up until the advent of probe microscopes, and some

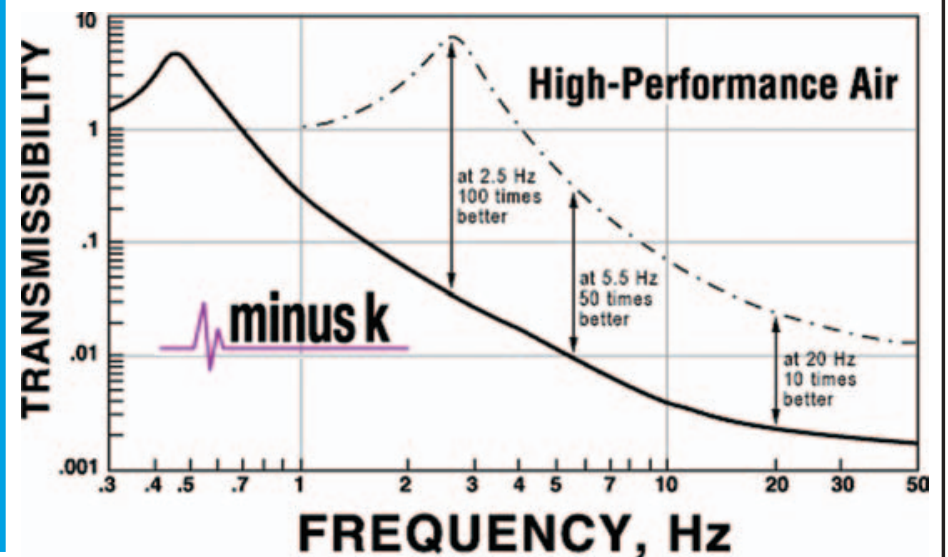

of the other very high-resolution imaging and data acquisition techniques, air isolators were adequate for most of applications. But not any longer.

What negative-stiffness isolators provide is really quite unique to the field of nanotechnology. In particular, the transmissibility of a negative-stiffness isolator-that is the vibration that transmits through the isolator as measured as a function of floor vibrations - which is substantially improved over air or active isolation systems. Although active isolation systems have fundamentally no resonance, their transmissibility does not roll off as fast as negative-stiffness isolators. So, at building and seismic frequencies the transmissibility of active isolators can be $10 \mathrm{X}$ greater than negative-stiffness isolators. This causes substantial adverse measurement and imaging artifacts in the data. Air isolators have the added disadvantage that their 2 to $2-1 / 2 \mathrm{~Hz}$ resonance affects a significant loss in isolation capability below about $5 \mathrm{~Hz}$. Negative-stiffness isolators are clearly the most efficient choice for probe microscopes.

Minus $\mathrm{K}^{\circledast}$ Technology, Inc. was founded in 1993 to develop, manufacture and market state-of-the-art vibration isolation products based on the company's patented negative-stiffness-mechanism technology. Minus $\mathrm{K}$ products, sold under the trade name Nano- $\mathrm{K}^{\circledast}$, are used in a broad spectrum of applications including nanotechnology, biological sciences, semiconductors, materials research, zero-g simulation of spacecraft, and high-end audio. The company is an OEM supplier to leading manufactures of scanning probe microscopes, micro-hardness testers and other vibrationsensitive instruments and equipment.

\section{Why have 1,000 scientists in $\mathbf{2 5}$ countries seleoted Minus $\mathbf{K}^{\circ}$ vibration isolators?}

Because Minus $\mathrm{K}$ vibration isolation systems deliver $10 x$ to $100 x$ better performance than high-performance air systems, without air, and for air table prices.

\section{Visit www.minusk.com for more information...}

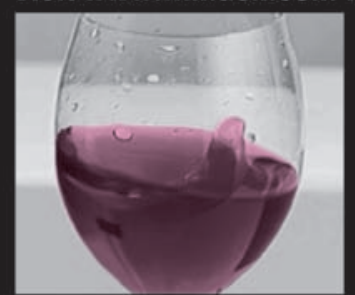

Without Minus K

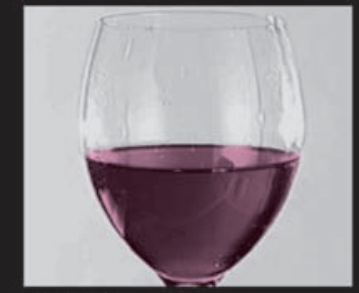

With Minus K
VIBRATION ISOLATION BY: minus $\mathbf{k}^{\circ}$ TECHNOLOGY

420 S. Hindry Ave., Unit E • Inglewood, CA 90301 USA Phone: 310-348-9656 • Fax: 310-348-9638 sales@minusk.com•www.minusk.com 